\title{
Balanced calibration of resonant piezoelectric RL shunts with quasi-static background flexibility correction
}

\author{
Høgsberg, Jan Becker; Krenk, Steen
}

Published in:

Journal of Sound and Vibration

Link to article, DOI:

10.1016/j.jsv.2014.12.006

Publication date:

2015

Document Version

Peer reviewed version

Link back to DTU Orbit

Citation (APA):

Høgsberg, J. B., \& Krenk, S. (2015). Balanced calibration of resonant piezoelectric $R L$ shunts with quasi-static background flexibility correction. Journal of Sound and Vibration, 341, 16-30.

https://doi.org/10.1016/j.jsv.2014.12.006

\section{General rights}

Copyright and moral rights for the publications made accessible in the public portal are retained by the authors and/or other copyright owners and it is a condition of accessing publications that users recognise and abide by the legal requirements associated with these rights.

- Users may download and print one copy of any publication from the public portal for the purpose of private study or research.

- You may not further distribute the material or use it for any profit-making activity or commercial gain

- You may freely distribute the URL identifying the publication in the public portal

If you believe that this document breaches copyright please contact us providing details, and we will remove access to the work immediately and investigate your claim. 


\title{
Balanced calibration of resonant piezoelectric $R L$ shunts with quasi-static background flexibility correction
}

\author{
Jan Høgsberg ${ }^{1}$ and Steen Krenk \\ Department of Mechanical Engineering, \\ Technical University of Denmark, DK-2800 Kongens Lyngby, Denmark \\ jhg@mek.dtu.dk , sk@mek.dtu.dk
}

\begin{abstract}
Resonant $R L$ shunt circuits constitute a robust approach to piezoelectric damping, where the performance with respect to damping of flexible structures requires a precise calibration of the corresponding circuit components. The balanced calibration procedure of the present paper is based on equal damping of the two modes associated with the resonant vibration form of the structure, when including a quasi-static contribution from non-resonant vibration modes via a single background flexibility parameter. Explicit calibration formulae are presented, and it is demonstrated by a numerical example that the procedure leads to equal modal damping and effective response reduction, even for rather indirect placement of the transducer, provided that the correction for background flexibility is included in the calibration procedure.
\end{abstract}

Keywords: $R L$ shunt circuit, Piezoelectric damping, Structural dynamics, Damper calibration, Background flexibility, Non-resonant modes

\section{Introduction}

Piezoelectric transducers are used in many different scientific and industrial applications for damping and control of the dynamic response of flexible structures $[1,2,3]$. Piezoelectric transducers are very versatile and may be used as for example actuator/sensor pairs in active control schemes $[3,4,5]$, as semi-active devices based on vari-

\footnotetext{
${ }^{1}$ Corresponding author: jhg@mek.dtu.dk
} 
ous switching techniques $[2,6]$, or as passive units in which the piezoelectric transducer is shunted to a suitable electric network $[7,8,9,10,11]$.

The passive damping concepts based on piezoelectric shunt circuits are attractive because they do not depend on an external power source and introduce no stability limits, although the compromise between performance and robustness naturally arises [1]. In the simplest shunt damping case the electrodes of the piezoelectric transducer are connected to a pure resistance $R$, which dissipates the electric energy converted from mechanical energy via the direct piezoelectric effect of the transducer [7, 12]. However, the attainable damping level associated with passive resistive shunt damping is limited by two circumstances: The limited magnitude of the electromechanical coupling coefficient and the inherent capacitance of the piezoelectric transducer, $[11,13,14,15]$. These limitations may be compensated by the introduction of a negative capacitor unit, which however yields an active element within the otherwise passive shunt circuit, $[16,17,18$, 19]. Alternatively, the effective damping may be increased for a particular vibration mode of the flexible structure by introducing resonant $R L$ shunt circuit damping, where an inductance $L$, together with the shunt resistance $R$ and the inherent capacitance of the piezoelectric, forms an additional resonance, $[7,8,9,10]$. This resonance can be used to increase the damping level for a targeted vibration mode, leading to a reduction of the corresponding vibration amplitudes, provided that the individual components of the $R L$ shunt circuit are calibrated appropriately, [20].

Several calibration concepts have been proposed for the components of series and parallel $R L$ shunt circuits. In Hagood and von Flotow [7] two different design concepts were proposed for the series $R L$ shunt circuit: A frequency response calibration, similar to that originally proposed by Brock [21] and Den Hartog [22] for the mechanical tuned mass absorber, and a pole-placement technique in which the modal damping ratio is determined by a root-locus analysis. A frequency response calibration for the parallel $R L$ shunt was subsequently proposed by $\mathrm{Wu}[9]$. A thorough summary of frequency response and pole-placement calibration techniques for both series and parallel $R L$ shunt circuits is given by Caruso [23], and in [17] the frequency response calibration of [7] has been used for $R L$ shunt circuits with an active negative capacitance unit to artificially increase the electromechanical coupling. Recently, the frequency response calibration 
from [7] has been modified slightly by Yamada et al. [24] to exactly follow the procedure used for classic mechanical tuned mass absorbers [22]. Alternatively, it has been demonstrated by Kim et al. [5] and Kozlowski et al. [25] that the governing equations of the piezoelectric $R L$ shunt circuits are analogous to those of slightly modified mechanical absorber configurations, and calibration expressions have been derived based on these modified models.

The original work by Hagood and von Flotow [7] demonstrates that for $R L$ shunt damping optimal frequency response calibration is in fact non-optimal with respect to a root-locus analysis, while optimal pole placement corresponds to non-optimal dynamic amplification properties. However, for the classic tuned mass absorber the fixed point frequency calibration is equivalent to equal damping in the two modes associated with the targeted vibration form, as demonstrated by Krenk [26]. Furthermore, the device damping is calibrated to provide an optimal combination of response reduction of the structure and limited absorber motion. Exact equivalence between equal modal damping and optimal response reduction appears to be a specific property of the mechanical tuned mass absorber, and is therefore not directly available for the present $R L$ shunt circuit formats. However, the property of equal modal damping, obtained by the proper frequency tuning of the mechanical absorber, is a desirable design property because it is independent of the particular loading and structural response, and therefore constitutes a robust design measure. As demonstrated in Krenk and Høgsberg [27] for general control formats and in Høgsberg and Krenk [28] for $R L$ shunt circuits the balanced calibration technique with equal modal damping also leads to proper reduction of the frequency response amplitudes for both the structural response and the damper force. Furthermore, the calibration procedure relies on a simple pole-placement technique, where the coefficients of the characteristic equation are matched with the coefficients of a generic polynomial. In the present paper this balanced calibration procedure is extended to include the effect of the flexibility associated with the non-resonant modes of the structure.

The performance of a resonant damping concept is often very sensitive to even a small detuning of the resonant filter coefficients and in particular the filter frequency. The calibration of resonant damping strategies typically relies on an approximate single 
mode representation of the flexible structure dynamics. For mechanical absorbers, as considered e.g. in $[22,26]$, this is often an adequate assumption because mechanical devices with an oscillating absorber mass operate with respect to absolute motion, and are typically located where the influence from other vibration modes is only moderate. This is different for piezoelectric transducers that act on the deformation of the structure, leading to positions at which the interaction with non-resonant residual vibration modes is typically larger. As the efficiency of resonant control systems depends on precise parameter calibration, the influence from residual non-resonant modes may be important for the performance of piezoelectric transducers. The inclusion of the influence from residual vibration modes by a quasi-static representation has previously been suggested for calibration of resonant active control strategies [32], and used for damping of edgewise vibrations of wind turbine blades [33] and rotors [34]. A more rigorous procedure for including the effect of the non-resonant modes as a local flexibility between the absorber and the structure was recently developed by Krenk and Høgsberg [35]. A similar approach is used in the present paper, leading to explicit formulae for equal modal damping calibration of the resonant $R L$ shunt circuit components, including a correction for the influence from non-resonant modes via a single background flexibility parameter.

\section{The electrical system}

Figure 1 shows the electric network representation of (a) a piezoelectric transducer, and the corresponding (b) series and (c) parallel configurations of a resonant $R L$ shunt circuit. For low-frequency applications a piezoelectric transducer can be represented by a time variable current source $i_{p}(t)$ connected in parallel to a capacitance $C$, [36], as indicated by the dashed box in Fig. 1a.

The current $i_{p}(t)$ is produced by straining of the piezoelectric transducer. The corresponding electric charge is $q_{p}(t)=-\theta u(t)$, where $\theta$ is the electromechanical coupling coefficient and $u(t)$ represents the deformation of the transducer. The charge in the capacitor element is $q_{c}(t)=C v(t)$, expressed in terms of the voltage $v(t)$ between the electrodes of the piezoelectric transducer, whereby $C$ represents the constant strain ca- 
(a)

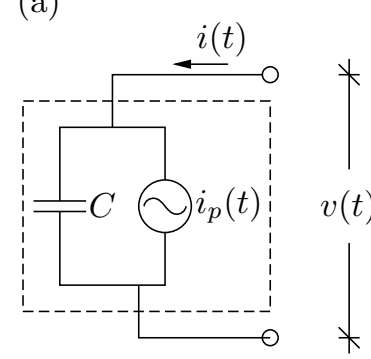

(b)

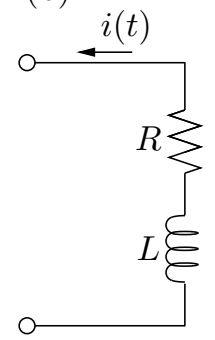

(c)

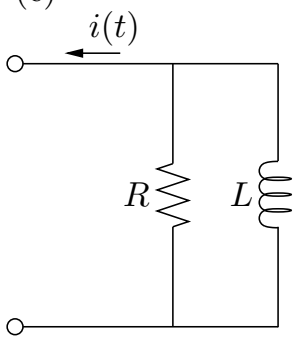

Figure 1: Electric model of (a) piezoelectric transducer, and (b) series or (c) parallel $R L$ shunt circuits.

pacitance associated with mechanically constrained transducer boundaries. The current flowing into the piezoelectric transducer is $i(t)=-\dot{q}(t)$, where the change in sign represents the opposite flow direction of current and rate of charge, and $\dot{(j)}=d() / d t$ denotes time differentiation. The current must be equal to the sum of the current produced by the current source and the current in the capacitor. When time integrating this electric current relation the following charge balance equation is readily obtained [4, 10, 23, 25],

$$
q(t)=-\theta u(t)+C v(t)
$$

where the two terms represent the individual contributions from the equivalent electric components in Fig. 1a. When the piezoelectric transducer is shunted with an electric $R L$ circuit the voltage $v(t)$ and charge $q(t)$ are related via the shunt circuit impedance, which together with (1) form a resonant filter that is able to effectively mitigate structural vibrations when the electric circuit components are calibrated appropriately.

\subsection{Series RL shunt}

Figure $1 \mathrm{~b}$ shows the series connection of the resistance $R$ and the inductance $L$, which forms the so-called series $R L$ shunt circuit. The electric current in the shunt circuit is $i(t)=-\dot{q}(t)$, and for the $R L$ shunt circuit this leads to the following relation between voltage and charge,

$$
v(t)=-(R \dot{q}(t)+L \ddot{q}(t))
$$

Elimination of $q(t)$ between (1) and (2) gives the resonant filter equation

$$
L C \ddot{v}(t)+R C \dot{v}(t)+v(t)=\theta(L \ddot{u}(t)+R \dot{u}(t))
$$


with acceleration and velocity feedback terms on the right hand side from the straining of the transducer. In normalized form this resonant filter equation can be written as

$$
\ddot{v}(t)+2 \zeta_{e} \omega_{e} \dot{v}(t)+\omega_{e}^{2} v(t)=\frac{\theta}{C}\left(\ddot{u}(t)+2 \zeta_{e} \omega_{e} \dot{u}(t)\right)
$$

in which the electric resonance frequency $\omega_{e}$ and damping ratio $\zeta_{e}$ for the series $R L$ shunt circuit have been introduced as

$$
\omega_{e}^{2}=\frac{1}{L C} \quad, \quad 2 \zeta_{e}=R \sqrt{\frac{C}{L}}
$$

The description of the properties and the parameter calibration procedure of resonant shunt circuits is most conveniently carried out in the frequency domain. In the present paper the time dependence is represented implicitly via the complex exponential factor $\mathrm{e}^{\mathrm{i} \omega t}$, where $\mathrm{i}=\sqrt{-1}$ is the complex imaginary unit and $\omega$ is the angular frequency. Thus, in the frequency domain representation time differentiation amounts to introduction of the factor $\mathrm{i} \omega$, and the impedance $Z$ of the shunt circuit is defined as

$$
v=Z i=-\mathrm{i} \omega Z q
$$

For the series $R L$ shunt circuit the impedance $Z$ follows from (2) as

$$
Z=R+\mathrm{i} \omega L
$$

In the resonant shunt circuit the inductance $L$ serves to define the undamped properties, while the resistance $R$ determines the damping. It may therefore be of interest to express the impedance in terms of $L$ and the damping ratio $\zeta_{e}$ at the filter resonance frequency $\omega_{e}$,

$$
Z_{e}=\mathrm{i} \omega_{e} L\left(1-\frac{\mathrm{i}}{\omega_{e}} \frac{R}{L}\right)=\mathrm{i} \omega_{e} L\left(1-2 \mathrm{i} \zeta_{e}\right)
$$

where the non-dimensional damping ratio $\zeta_{e}$ has been introduced from $(5 \mathrm{~b})$. It is seen from the last factor that the role of the damping parameter $2 \zeta_{e}$ is to change the phase of the impedance. In typical damping applications the damping ratio will be in the order of a few percent. Thus, the relation in (8) indicates that in the series coupled circuit in Fig. 1 b the resistance is typically limited by $R \ll \sqrt{C / L}$. The physical explanation is that the resistance should only moderately reduce the current through the inductor $L$. 


\subsection{Parallel RL shunt}

The parallel $R L$ shunt circuit is shown in Fig. 1c, and for this parallel configuration the relation between voltage and charge is

$$
\frac{1}{R} \dot{v}(t)+\frac{1}{L} v(t)=-\ddot{q}(t)
$$

Elimination of $q(t)$ between (1) and (9) gives the resonant filter equation,

$$
C \ddot{v}(t)+\frac{1}{R} \dot{v}(t)+\frac{1}{L} v(t)=\theta \ddot{u}(t)
$$

Thus, for the parallel $R L$ shunt circuit the resonant filter receives pure acceleration feedback from the straining of the piezoelectric transducer. This filter equation is normalized, whereby it takes the form

$$
\ddot{v}(t)+2 \zeta_{e} \omega_{e} \dot{v}(t)+\omega_{e}^{2} v(t)=\frac{\theta}{C} \ddot{u}(t)
$$

In this normalized equation the frequency and damping parameters are defined by

$$
\omega_{e}^{2}=\frac{1}{L C} \quad, \quad 2 \zeta_{e}=\frac{1}{R} \sqrt{\frac{L}{C}}
$$

It is seen that the circuit resonance frequency $\omega_{e}$ is the same as for the series system in (5), while the damping parameter $2 \zeta_{e}$ is given by the reciprocal of the parameter combination for the series circuit. In the case of the parallel shunt circuit the impedance $Z$ follows from the frequency domain representation of (9),

$$
\frac{1}{Z}=\frac{1}{R}+\frac{1}{\mathrm{i} \omega L}
$$

At the resonance frequency $\omega_{e}$ the impedance can be written in the form

$$
Z_{e}=\frac{\mathrm{i} \omega_{e} L}{1+\frac{\mathrm{i} \omega_{e} L}{R}}=\frac{\mathrm{i} \omega_{e} L}{1+2 \mathrm{i} \zeta_{e}}
$$

It is seen from the denominator that again the role of the damping parameter $2 \zeta_{e}$ is to change the phase of the impedance, and for a damping ratio of a few percent the resistance is typically limited by $R \gg \sqrt{C / L}$, indicating only moderate current through the resistance branch of the parallel shunt circuit in Fig. 1c. 


\section{The mechanical system}

The piezoelectric transducer of the previous section is now attached to a flexible structure. The purpose of the $R L$ shunt circuit is to introduce a supplemental dynamic resonance to the system, which is to be designed to effectively reduce excessive amplification of the dynamic response around the natural frequency of the structure. The performance of a resonant damping strategy relies on the precise calibration of the individual system components, and in the case of a flexible structure the resonant vibration properties used for the shunt circuit parameter calibration in Section 5 must be represented in terms of the modal properties of the structure. The present section describes a simple procedure, by which the modal properties of the structure are condensed into the mass and stiffness of the resonant vibration mode, while the background deformation from the remaining non-resonant modes is represented in an approximate form via a single flexibility parameter.

\subsection{Structure with piezoelectric transducer}

The dynamics of the flexible structure is represented by a discrete numerical model with the equation of motion

$$
\mathbf{M} \ddot{\mathbf{u}}(t)+\mathbf{K u}(t)=-\mathbf{w} \theta v(t)+\mathbf{f}_{e}(t)
$$

In this equation the column vector $\mathbf{u}(t)$ contains the degrees of freedom of the numerical model, the mass matrix $\mathbf{M}$ and stiffness matrix $\mathbf{K}$ represent the inertia and elastic properties of the combined structure, while the column vector $\mathbf{f}_{e}(t)$ represents the external loading on the structure. The first term on the right hand side of (15) represents the electromechanical force on the structure from the piezoelectric transducer, where the connectivity vector $\mathbf{w}$ defines the deformation of the transducer

$$
u(t)=\mathbf{w}^{T} \mathbf{u}(t)
$$

and the electromechanical coupling coefficient $\theta$ appears as a proportionality factor. It is seen that this electromechanical forcing term in (15) vanishes for $v(t)=0$, and the stiffness matrix $\mathbf{K}$ in (15) therefore represents the elastic stiffness of the structure with the piezoelectric transducer attached with short-circuited electrodes, as shown in Fig. 2a. 
(a)

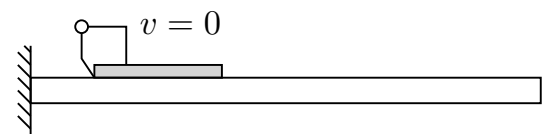

(b)

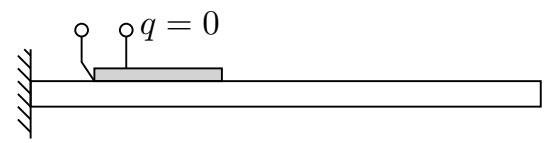

Figure 2: Flexible structure with transducer in (a) short- and (b) open-circuit conditions.

The voltage $v(t)$ in (15) may be eliminated by the charge balance equation (1), which leads to the alternative form of the equation of motion

$$
\mathbf{M} \ddot{\mathbf{u}}(t)+\left(\mathbf{K}+\frac{\theta^{2}}{C} \mathbf{w} \mathbf{w}^{T}\right) \mathbf{u}(t)=-\mathbf{w} \frac{\theta}{C} q(t)+\mathbf{f}_{e}(t)
$$

where the electromechanical force is now given by the charge $q(t)$. This form of the equation of motion introduces the modified stiffness matrix $\mathbf{K}+\left(\theta^{2} / C\right) \mathbf{w} \mathbf{w}^{T}$. Because the electromechanical force in (17) vanishes for $q(t)=0$ this modified stiffness governs the system properties associated with open-circuit electrode conditions, see Fig. 2b.

For the calibration and design of shunt circuit concepts the flexible structure is typically represented by a single vibration form, commonly obtained from the eigenvalue problem associated with $v(t)=0$ in (15). However, because the shunt circuit impedance (6) defines the voltage as function of charge, the preferred structural equation of motion is often that in (17). For an assumed single mode representation the additional stiffness term in (17) appears in scalar form and represents the apparent change in natural frequency between the short- and open-circuit conditions. The purpose of the present paper is to demonstrate how the influence from residual vibration modes can be accurately represented by a single background flexibility parameter, and in this connection it is advantageous to address the equation of motion in (15), where the stiffness matrix $\mathbf{K}$ only contains elastic contributions.

\subsection{Equivalent structural parameters}

The structural properties, including the effect of non-resonant modes, are determined according to the procedure developed in Krenk and Høgsberg [35]. The governing equa- 
tions of motion in (15) may be written in the general form

$$
\mathbf{M} \ddot{\mathbf{u}}(t)+\mathbf{K u}(t)=\mathbf{w} f(t)+\mathbf{f}_{e}(t)
$$

where the electromechanical force $f(t)=-\theta v(t)$ is proportional to the voltage across the transducer electrodes. The structural parameters, including a quasi-static contribution from the non-resonant modes, are conveniently identified from the frequency representation of (18), obtained by introducing the harmonic exponential representations $\mathbf{u}(t)=\mathbf{u e}^{\mathrm{i} \omega t}$ and $f(t)=f \mathrm{e}^{\mathrm{i} \omega t}$ with angular frequency $\omega$ and amplitudes $\mathbf{u}$ and $f$. Substitution into (18) gives the following frequency equation, which in the absence of external forces reads

$$
\left[\mathbf{K}-\omega^{2} \mathbf{M}\right] \mathbf{u}=\mathbf{w} f
$$

The formal solution for the displacement vector $\mathbf{u}$ follows by inversion of the dynamic stiffness matrix, and the scalar transducer deformation $u$ is then given by

$$
u=\mathbf{w}^{T} \mathbf{u}=\left(\mathbf{w}^{T}\left[\mathbf{K}-\omega^{2} \mathbf{M}\right]^{-1} \mathbf{w}\right) f
$$

The frequency dependent term in the parenthesis is the dynamic flexibility of the structure at the location of the piezoelectric force $f$, and the purpose of the following analysis is to derive a simple representation in the form of a resonant term and an additional quasi-static term, accounting for background flexibility. The quasi-static representation of high-frequency vibration modes is a well-established truncation technique in the numerical analysis of structures $[29,30]$ and for accurate prediction of the system poles in structural control $[3,31]$.

The inverse of the system matrix $\left[\mathbf{K}-\omega^{2} \mathbf{M}\right]$ can be represented in terms of the eigenvectors $\mathbf{u}_{1}, \cdots, \mathbf{u}_{n}$ and eigenfrequencies $\omega_{1}, \cdots, \omega_{n}$ of the corresponding generalized eigenvalue problem in (19) with $f=0$,

$$
\left[\mathbf{K}-\omega^{2} \mathbf{M}\right]^{-1}=\sum_{j=1}^{n} \frac{\omega_{j}^{2}}{\omega_{j}^{2}-\omega^{2}} \frac{\mathbf{u}_{j} \mathbf{u}_{j}^{T}}{\mathbf{u}_{j}^{T} \mathbf{K} \mathbf{u}_{j}} .
$$

This result follows from an eigenvector representation of the displacement and force vector of the dynamic problem (19). The dynamic flexibility at the transducer location 
as given in (20) then follows in the form

$$
\mathbf{w}^{T}\left[\mathbf{K}-\omega^{2} \mathbf{M}\right]^{-1} \mathbf{w}=\sum_{j=1}^{n} \frac{\omega_{j}^{2}}{\omega_{j}^{2}-\omega^{2}} \frac{1}{k_{j}} \simeq \frac{\omega_{r}^{2}}{\omega_{r}^{2}-\omega^{2}} \frac{1}{k_{r}}+\sum_{j \neq r}^{n} \frac{1}{k_{j}}
$$

where the parameter $k_{j}$ is the modal stiffness associated with the mode shape vector $\mathbf{u}_{j} /\left(\mathbf{w}^{T} \mathbf{u}_{j}\right)$, normalized to unity over the transducer,

$$
k_{j}=\frac{\mathbf{u}_{j}^{T} \mathbf{K} \mathbf{u}_{j}}{\left(\mathbf{w}^{T} \mathbf{u}_{j}\right)^{2}}
$$

In the last expression in (22) only the resonant term with $j=r$ is retained in its frequency-dependent form, while the remaining non-resonant terms are replaced by their equivalent quasi-static form. The last sum, representing the background flexibility from the non-resonant modes, can be expressed directly by considering the expansion in (22) for $\omega=0$,

$$
\mathbf{w}^{T} \mathbf{K}^{-1} \mathbf{w}=\sum_{j=1}^{n} \frac{1}{k_{j}}=\frac{1}{k_{r}}+\sum_{j \neq r}^{n} \frac{1}{k_{j}}=\frac{1}{k_{r}}+\frac{1}{k_{0}}
$$

Here the term $1 / k_{0}$ represents the background flexibility, formed by the sum of the quasistatic flexibility of all the non-resonant modes. It is noted that the background flexibility $1 / k_{0}$ can be evaluated directly from (24) using only the inverse of the stiffness matrix and the stiffness $k_{r}$ of the resonant mode, but without evaluating dynamic properties of any of the non-resonant modes. When introducing the approximate dynamic flexibility (22) into the scalar structure equation (20), the following relation is obtained for the deformation of the transducer

$$
u \simeq\left[\frac{\omega_{r}^{2}}{\omega_{r}^{2}-\omega^{2}} \frac{1}{k_{r}}+\frac{1}{k_{0}}\right] f
$$

It is important to note that this relation connects the local deformation $u$ of the piezoelectric transducer and the corresponding local piezoelectric force $f$. These quantities describe the response of the piezoelectric device, while the response of the structure is described by a classic modal analysis, as discussed in the next section.

\subsection{Modified modal equations}

The expression (25) gives the key to the approximate inclusion of the effect of nonresonant background modes. The local deformation $u(t)$ is expressed as the sum of a 
resonant modal deformation $u_{r}(t)$ and an additional quasi-static term $f(t) / k_{0}$, corresponding to the representation

$$
u(t)=u_{r}(t)+\frac{1}{k_{0}} f(t)
$$

where $u_{r}(t)$ follows from the classic modal equation

$$
m_{r} \ddot{u}_{r}(t)+k_{r} u_{r}(t)=f(t)+f_{r}(t)
$$

corresponding to the normalized mode shape vector $\mathbf{u}_{r} /\left(\mathbf{w}^{T} \mathbf{u}_{r}\right)$. In this relation $m_{r}$ is the modal mass, while $f_{r}(t)$ is the modal component of the external load,

$$
m_{r}=\frac{\mathbf{u}_{r}^{T} \mathbf{M} \mathbf{u}_{r}}{\left(\mathbf{w}^{T} \mathbf{u}_{r}\right)^{2}} \quad, \quad f_{r}(t)=\frac{\mathbf{u}_{r}^{T}}{\mathbf{w}^{T} \mathbf{u}_{r}} \mathbf{f}_{e}(t)
$$

In (27) the piezoelectric force $f(t)$ also constitutes the corresponding modal force due to the normalization of the resonant mode. The modal mass is related to the modal stiffness defined in (23) by $k_{r}=\omega_{r}^{2} m_{r}$. For the series and parallel $R L$ shunt circuits the structural modal equation (27) is combined with the local equations in (4) and (11), respectively. The corresponding transducer deformation $u(t)$ is eliminated by (26) with the piezoelectric force defined as $f(t)=-\theta v(t)$.

\section{Complex root analysis}

The calibration procedure of the present paper is based on combining the selected resonant mode with the appropriate resonant filter from the $R L$ shunt circuit. These are governed by two second-order dynamic equations, one for the modal response and one for the resonant shunt. Thus, the dynamics of the combined system is described by a characteristic equation of degree four in the natural frequency $\omega$. The calibration is based on the principle of equal damping of the two modes generated by the electromechanical coupling with the resonant mode of the structure. This is a pole placement procedure, developed for the tuned mass damper in [26] and extended to general control formats in $[27,28]$. The present section constructs the generic polynomial equation with equal modal damping properties, and the calibration procedure then follows directly from parameter equivalence, as described in Section 5 . 
Let the four roots of the characteristic equation be denoted $\omega_{1}, \cdots, \omega_{4}$, and assume that there is a parameter combination for which $\omega_{1}$ and $\omega_{2}$ lie in the first quadrant of the complex plane. The corresponding vibration modes will then have equal damping ratio, if $\omega_{1}$ and $\omega_{2}$ lie on the same line containing the origin of the complex plane, as illustrated in Fig. 3. This implies that the roots $\omega_{1}$ and $\omega_{2}$ are inverse points with respect to a circle with radius $\omega_{0}$. This inverse point property can be expressed as

$$
\frac{\omega_{2}}{\omega_{0}}=\frac{\omega_{0}}{\omega_{1}^{*}}
$$

where $\omega_{1}^{*}$ denotes the complex conjugate of $\omega_{1}$. The reciprocal relation in (29) between $\omega / \omega_{0}$ and $\omega_{0} / \omega$ suggests the following format of the characteristic equation,

$$
\left(\frac{\omega}{\omega_{0}}-\frac{\omega_{0}}{\omega}\right)^{2}-4 i \lambda \xi\left(\frac{\omega}{\omega_{0}}-\frac{\omega_{0}}{\omega}\right)-4 \xi^{2}=0
$$

Details concerning the construction and form of this characteristic equation can be found in reference [26]. The above equation contains two coefficients, and these are conveniently expressed in terms of the two real-valued parameters $\xi$ and $\lambda$, as shown in (30). The corresponding polynomial form follows from multiplication with $\left(\omega_{0} \omega\right)^{2}$,

$$
\omega^{4}-\left(2+4 \xi^{2}\right) \omega_{0}^{2} \omega^{2}+\omega_{0}^{4}-4 i \lambda \xi \omega_{0} \omega\left(\omega^{2}-\omega_{0}^{2}\right)=0
$$

It is seen that the reference frequency $\omega_{0}$ is defined by the constant term of the normalized characteristic polynomial. The special property of equally damped modes, as expressed by the inverse point relation (29), is equivalent to imposing a balance between the cubic and linear terms, whereby they cancel at the reference frequency $\omega= \pm \omega_{0}$.

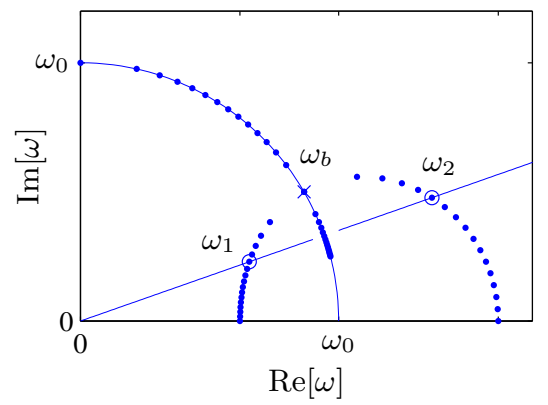

Figure 3: Root locus diagram for generic polynomial with circles indicating roots $\omega_{1}, \omega_{2}$ for $\lambda=\frac{1}{2} \sqrt{2}$. 
The root locus diagram of the quartic equation (31) is easily analyzed by reverting to the quadratic format (30). This format permits determination of the expression inside the parenthesis, and $\omega$ then follows from solving a quadratic equation, see [26] for details. The results are illustrated in Fig. 3. For $\lambda=0$ there is no damping because the second term in (30) vanishes, and the natural frequencies $\omega_{1}$ and $\omega_{2}$ appear as points on the positive real axis. When increasing $\lambda$ for a fixed value of the parameter $\xi<1$, the roots move into the complex plane as illustrated in Fig. 3. It follows from the inverse point property (29), illustrated by the straight line in Fig. 3, that the two roots $\omega_{1}$ and $\omega_{2}$ have equal argument, corresponding to equal damping ratio of the associated free vibration modes. This situation changes at the bifurcation point $\omega_{b}$, which is reached for $\lambda=1$. The roots then branch off along a circle with radius $|\omega|=\omega_{0}$. One branch reaches a new branch point $\omega=\mathrm{i} \omega_{0}$ on the imaginary axis, while the other root follows the circle towards $\omega=\omega_{0}$ on the real axis.

The parameter values of interest in connection with control and damping of structures correspond to the first part where the roots $\omega_{1}$ and $\omega_{2}$ are inverse points in the circle, corresponding to the parameter interval $0<\lambda \leq 1$. In this parameter interval the two complex roots have the form

$$
\omega_{1,2}=\left|\omega_{1,2}\right|\left(\sqrt{1-\zeta^{2}}+i \zeta\right)
$$

where $\zeta$ is the common damping ratio of the two vibration modes associated with the targeted resonance of the structure. A simple expression for the modal damping ratio $\zeta$ in terms of the system parameters $\lambda$ and $\xi$ can be obtained by using the fact that the coefficient of the cubic term in (31) is the sum of the four roots $\omega_{1}, \cdots, \omega_{4}$. When using the special symmetry properties of the four roots the following expression is obtained for the damping ratio

$$
\zeta=\frac{\lambda \xi}{\frac{1}{2}\left(\frac{\left|\omega_{1}\right|}{\omega_{0}}+\frac{\omega_{0}}{\left|\omega_{1}\right|}\right)} \simeq \lambda \xi
$$

where the latter approximation is valid for small values of $\xi$, typical of practice.

At first it may appear desirable to choose $\lambda=1$ corresponding to the bifurcation point $\omega_{b}$, as this introduces maximum damping ratio in the modes, $[7,23]$. However, this also implies identical angular frequency of the modes $\left(\omega_{1}=\omega_{2}\right)$, which leads to 
constructive interference of the two vibration forms and thereby an undesirable single peak in the dynamic response amplification, as illustrated in $[7,26]$. As demonstrated in [26] an optimal balance between the attained damping ratio and a suitable separation of the vibration frequencies is obtained for the parameter value $\lambda=\frac{1}{2} \sqrt{2}$. For small to moderate damping ratios the two roots $\omega_{1}$ and $\omega_{2}$ are then located at points on the near semi-circular contour at an angle of $\pm 45^{\circ}$ with the real axis. This leads to the following approximate, but quite accurate, expression for the modal damping ratio

$$
\zeta \simeq \frac{1}{2} \sqrt{2} \xi
$$

In the calibration procedure described in the next section the damping ratio $\zeta$ resulting from the application of the piezoelectric transducer is used as a selected design input parameter.

\section{Calibration of resonant shunt circuits}

The calibration procedure is formulated in terms of the modal response $u_{r}(t)$ described by the modal equation of motion derived from (27),

$$
\ddot{u}_{r}(t)+\omega_{r}^{2} u_{r}(t)=\omega_{r}^{2} \frac{1}{k_{r}} f(t)+\omega_{r}^{2} \frac{1}{k_{r}} f_{r}(t)
$$

where $f(t)=-\theta v(t)$ is the local force generated by the piezoelectric transducer. This modal equation in terms of the modal displacement $u_{r}(t)$ is complemented by a local equation for the transducer force $f(t)$, governed by feedback described in terms of the local deformation of the transducer $u(t)$. The modal and local displacements are related by the equation (26), which includes the effect of the background flexibility via the term $f(t) / k_{0}$.

\subsection{Series $R L$ shunt}

In the case of the series $R L$ shunt circuit the governing equation (4) relates the voltage $v(t)$ and the local deformation of the transducer $u(t)$. When $u(t)$ is expressed in terms of the modal displacement $u_{r}(t)$ by $(26)$ and the transducer force is introduced by 
the relation $f(t)=-\theta v(t)$, the following second order differential equation is obtained

$$
\begin{aligned}
\ddot{f}(t)+2 \zeta_{e} \omega_{e} \dot{f}(t)+\omega_{e}^{2} f(t)+\frac{\theta^{2}}{C}\left[\ddot{u}_{r}(t)+2 \zeta_{e} \omega_{e} \dot{u}_{r}(t)\right. \\
\left.+\frac{1}{k_{0}}\left(\ddot{f}(t)+2 \zeta_{e} \omega_{e} \dot{f}(t)\right)\right]=0
\end{aligned}
$$

The coefficient of the force term inside the square brackets suggests the introduction of non-dimensional parameters expressing the electromechanical coupling relative to the modal stiffness and the background stiffness, respectively,

$$
\kappa_{r}=\frac{\theta^{2}}{C k_{r}} \quad, \quad \kappa_{0}=\frac{\theta^{2}}{C k_{0}}
$$

It is noted that the coefficient $\kappa_{r}$ represents the square of the generalized coupling coefficient with respect to the mode $r$. This parameter is commonly estimated - without background flexibility correction - via the relative difference between the square of the open and short circuit natural frequencies, see for example [7, 20, 37].

After collecting the force terms and division by the modal stiffness $k_{r}$ the equation (36) takes the convenient form

$$
\frac{1}{k_{r}}\left(\ddot{f}(t)+2 \zeta_{e} \omega_{e} \dot{f}(t)+\frac{\omega_{e}^{2}}{1+\kappa_{0}} f(t)\right)+\frac{\kappa_{r}}{1+\kappa_{0}}\left(\ddot{u}_{r}(t)+2 \zeta_{e} \omega_{e} \dot{u}_{r}(t)\right)=0
$$

The dynamic equations (35) and (38) are expressed in the frequency domain by introduction of the complex representation $u_{r}(t)=u_{r} \mathrm{e}^{\mathrm{i} \omega t}$ and $f(t)=f \mathrm{e}^{\mathrm{i} \omega t}$, and then combined into the compact matrix format

$$
\left[\begin{array}{cc}
\omega_{r}^{2}-\omega^{2} & -\omega_{r}^{2} \\
\frac{\kappa_{r}}{1+\kappa_{0}}\left(-\omega^{2}+2 \mathrm{i} \zeta_{e} \omega_{e} \omega\right) & \frac{\omega_{e}^{2}}{1+\kappa_{0}}-\omega^{2}+2 \mathrm{i} \zeta_{e} \omega_{e} \omega
\end{array}\right]\left[\begin{array}{c}
u_{r} \\
f / k_{r}
\end{array}\right]=\left[\begin{array}{c}
\omega_{r}^{2} f_{r} / k_{r} \\
0
\end{array}\right]
$$

The natural vibration frequencies $\omega$ corresponding to these equations follow from the characteristic equation

$$
\begin{aligned}
\omega^{4}-\left(\left(1+\kappa_{r}+\kappa_{0}\right) \omega_{r}^{2}+\omega_{e}^{2}\right) \frac{\omega^{2}}{1+\kappa_{0}}+\frac{\omega_{e}^{2} \omega_{r}^{2}}{1+\kappa_{0}} & \\
- & 2 \mathrm{i} \zeta_{e} \omega_{e} \omega\left(\omega^{2}-\frac{1+\kappa_{r}+\kappa_{0}}{1+\kappa_{0}} \omega_{r}^{2}\right)=0
\end{aligned}
$$

The calibration procedure consists in reducing the actual characteristic equation (40) to the particular form of the generic equation in (31) with the equal modal damping properties illustrated in Fig. 3. 
The first step in the calibration procedure is to identify the reference frequency $\omega_{0}$ from the coefficients to the linear and the cubic terms. This gives

$$
\omega_{0}^{2}=\frac{1+\kappa_{r}+\kappa_{0}}{1+\kappa_{0}} \omega_{r}^{2}
$$

The reference frequency $\omega_{0}$ is also defined by the constant term of the characteristic equation, which yields the relation

$$
\omega_{0}^{4}=\frac{\omega_{e}^{2} \omega_{r}^{2}}{1+\kappa_{0}}
$$

Elimination of $\omega_{0}$ between (41) and (42) then determines the shunt circuit frequency as

$$
\omega_{e}^{2}=\frac{\left(1+\kappa_{r}+\kappa_{0}\right)^{2}}{1+\kappa_{0}} \omega_{r}^{2}
$$

It is seen from this relation that the shunt circuit frequency $\omega_{e}$ is always larger than the resonant modal frequency $\omega_{r}$ of the structure. This is different from the mechanical tuned mass absorber, for which the tuning frequency of the absorber is lower than the natural frequency of the structure in the absence of background flexibility, but increases with increasing background flexibility and may become larger than the structural frequency, $[26,35]$.

The second step in the calibration procedure determines the damping property of the shunt circuit, represented by the damping parameter $\zeta_{e}$. First $\zeta_{e}$ is determined in terms of the generic parameter $\xi$ by comparing the coefficients of the cubic terms in (31) and (40). For $\lambda=\frac{1}{2} \sqrt{2}$ this gives

$$
\zeta_{e}^{2}=2 \xi^{2} \frac{\omega_{0}^{2}}{\omega_{e}^{2}}
$$

The parameter $\xi$ also follows by comparing the coefficients of the quadratic terms, and when using the frequency solutions in (41) and (43) the following expression is obtained,

$$
4 \xi^{2}=\frac{\kappa_{r}}{1+\kappa_{0}}
$$

Elimination of $\xi$ between (44) and (45) determines the optimal shunt circuit damping parameter as

$$
\zeta_{e}^{2}=\frac{1}{2} \frac{\kappa_{r}}{\left(1+\kappa_{0}\right)\left(1+\kappa_{r}+\kappa_{0}\right)}
$$


An estimate of the corresponding modal damping ratio $\zeta$ is obtained by elimination of the generic parameter $\xi$ between (45) and (34),

$$
\zeta^{2} \simeq \frac{1}{8} \frac{\kappa_{r}}{1+\kappa_{0}}
$$

It is seen that the effect of the background flexibility, represented by the non-dimensional parameter $\kappa_{0}$, is to reduce the damping attained for a given coupling parameter $\kappa_{r}$.

\subsection{Parallel RL shunt}

In the case of the parallel $R L$ shunt circuit the voltage $v(t)$ is governed by the equation (11), where $v(t)$ is then replaced by the transducer force $f(t)=-\theta v(t)$ and the local transducer deformation $u(t)$ is eliminated by the relation (26). This gives the differential equation

$$
\ddot{f}(t)+2 \zeta_{e} \omega_{e} \dot{f}(t)+\omega_{e}^{2} f(t)+\frac{\theta^{2}}{C}\left[\ddot{u}_{r}(t)+\frac{1}{k_{0}} \ddot{f}(t)\right]=0
$$

which is similar to that of the series $R L$ shunt in (36), but without the first derivatives inside the square brackets. As before, collecting the force terms and dividing by the modal stiffness leads to the transducer equation

$$
\frac{1}{k_{r}}\left(\ddot{f}(t)+\frac{2 \zeta_{e} \omega_{e}}{1+\kappa_{0}} \dot{f}(t)+\frac{\omega_{e}^{2}}{1+\kappa_{0}} f(t)\right)+\frac{\kappa_{r}}{1+\kappa_{0}} \ddot{u}_{r}(t)=0
$$

In this case combination of the frequency equations corresponding to (35) and (49) are combined into the matrix format

$$
\left[\begin{array}{cc}
\omega_{r}^{2}-\omega^{2} & -\omega_{r}^{2} \\
-\frac{\kappa_{r}}{1+\kappa_{0}} \omega^{2} & \frac{\omega_{e}^{2}}{1+\kappa_{0}}-\omega^{2}+\frac{2 \mathrm{i} \zeta_{e} \omega_{e}}{1+\kappa_{0}} \omega
\end{array}\right]\left[\begin{array}{c}
u_{r} \\
f / k_{r}
\end{array}\right]=\left[\begin{array}{c}
\omega_{r}^{2} f_{r} / k_{r} \\
0
\end{array}\right]
$$

which leads to the following fourth degree characteristic equation in $\omega$,

$$
\omega^{4}-\left(\left(1+\kappa_{r}+\kappa_{0}\right) \omega_{r}^{2}+\omega_{e}^{2}\right) \frac{\omega^{2}}{1+\kappa_{0}}+\frac{\omega_{e}^{2} \omega_{r}^{2}}{1+\kappa_{0}}-\frac{2 \mathrm{i} \zeta_{e} \omega_{e}}{1+\kappa_{0}} \omega\left(\omega^{2}-\omega_{r}^{2}\right)=0
$$

This equation is rather similar to (40) for the case of a series $R L$ shunt circuit, the only difference occuring in the last term.

The balance between the linear and the cubic terms immediately identifies the reference frequency $\omega_{0}=\omega_{r}$. The relation for the constant term is given by (42), as for the 
series $R L$ shunt, and thus the transducer frequency follows directly as

$$
\omega_{e}^{2}=\left(1+\kappa_{0}\right) \omega_{r}^{2}
$$

This shows that for the parallel connection the shunt circuit frequency $\omega_{e}$ is also larger than the resonant modal frequency $\omega_{r}$ in this case, but only when including the effect of background flexibility.

The damping parameter $\zeta_{e}$ is given in terms of $\xi$ by comparing the coefficients of the cubic term,

$$
\zeta_{e}^{2}=2 \xi^{2} \frac{\omega_{0}^{2}}{\omega_{e}^{2}}\left(1+\kappa_{0}\right)^{2}=2 \xi^{2}\left(1+\kappa_{0}\right)
$$

The generic parameter $\xi$ follows by comparing the coefficients of the quadratic terms, and when using $\omega_{0}=\omega_{r}$ and (52) the expression (45) is obtained, also in this case. Substitution of this result into (53) then gives the optimal damping parameter

$$
\zeta_{e}^{2}=\frac{1}{2} \kappa_{r}
$$

Finally, the modal damping ratio follows from the generic formula (34) as

$$
\zeta^{2} \simeq \frac{1}{8} \frac{\kappa_{r}}{1+\kappa_{0}}
$$

Thus, the attainable modal damping introduced by an optimally calibrated parallel $R L$ shunt is identical to that obtained from an optimally calibrated series $R L$ shunt, in spite of the fact that the optimal calibration leads to different shunt circuit frequency $\omega_{e}$ and shunt damping parameter $\zeta_{e}$.

\subsubsection{Calibration procedure}

The design formulae derived above are given in a format where the electromechanical properties, comprised by the parameter $\theta^{2} / C$, are assumed known, and the shunt circuit characteristics and attainable modal damping are then calculated. However, it is desirable to have a design procedure in which the vibration properties, characterized by the resulting modal damping ratio $\zeta$ of the targeted resonant mode, is given together with the structural properties, and the procedure is therefore reversed to obtain an optimal value of the electromechanical parameter $\theta^{2} / C$, and subsequently the inductance $L$ and resistance $R$, from a desired modal damping ratio. 
Table 1: Calibration of $R L$ shunt circuit components.

\begin{tabular}{lcc}
\hline & $L C \omega_{r}^{2}$ & $R C \omega_{r}$ \\
\hline series: & $\frac{1+\kappa_{0}}{\left(1+\kappa_{0}+\kappa_{r}\right)^{2}}$ & $\sqrt{\frac{2 \kappa_{r}}{\left(1+\kappa_{0}+\kappa_{r}\right)^{3}}}$ \\
parallel: & $\frac{1}{1+\kappa_{0}}$ & $\sqrt{\frac{1}{2 \kappa_{r}\left(1+\kappa_{0}\right)}}$ \\
\hline
\end{tabular}

Therefore, let the desired modal damping ratio $\zeta$ be given. When the background flexibility coefficient $\kappa_{0}$ is expressed in terms of the modal flexibility coefficient $\kappa_{r}$ as $\kappa_{0}=\left(k_{r} / k_{0}\right) \kappa_{r}$, the damping formulae (47) and (55), that are identical for the series and parallel shunts, can be inverted to give

$$
\kappa_{r}=\frac{8 \zeta^{2}}{1-8\left(k_{r} / k_{0}\right) \zeta^{2}}
$$

The relation (37a) then gives the electromechanical coupling parameter as

$$
\frac{\theta^{2}}{C}=\kappa_{r} k_{r}
$$

while the corresponding shunt circuit components $L$ and $R$ are determined by the design expressions in Table 1. It is seen that the introduction of background flexibility, important for a more indirect location of the piezoelectric transducer on the structure, leads to a larger electromechanical coupling coefficient.

Figure 4 shows the dynamic amplification and the frequency amplitude of the piezoelectric force for a single mode structure obtained from the frequency expressions in (39) and (50) with $\kappa_{0}=0$. For both of the desired damping ratios $\zeta=0.02$ and 0.04 the figure shows two curves, representing the series (solid curve) and the parallel (dashed curve) $R L$ shunt circuit, respectively. It is seen in the figure that the $R L$ shunt circuit damping with balanced calibration based on equal modal damping leads to an effective response reduction, with slightly improved performance obtained by the parallel shunt circuit. Furthermore, Fig. 4b shows that the balanced calibration leads to an ideally flat plateau for the transducer force amplitude around the resonance frequency, while an 

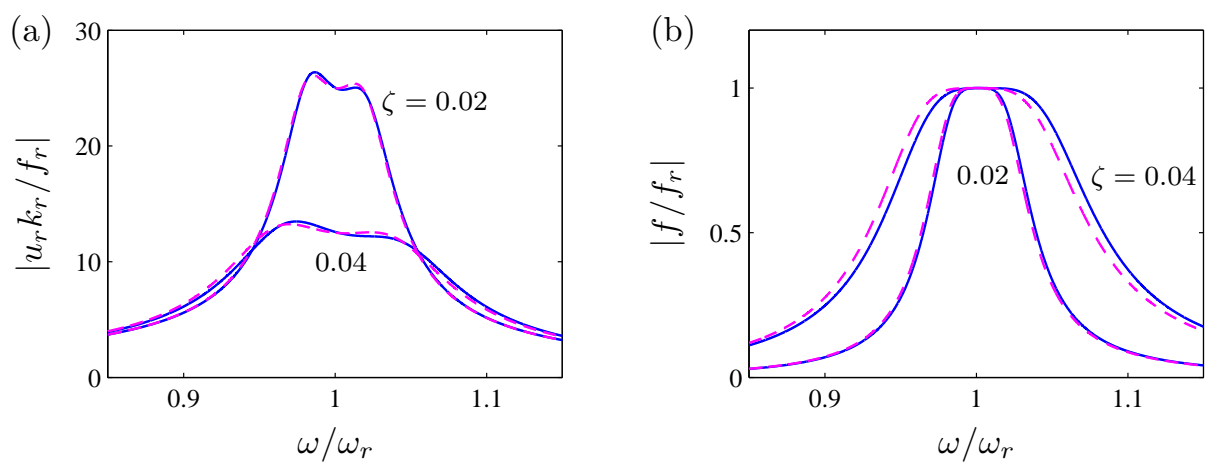

Figure 4: (a) Dynamic amplification and (b) force amplitude with $\zeta=0.02$ and 0.04: Series $(-$ ) and parallel (-- $R L$ shunt circuit.

increase in the desired damping ratio yields a corresponding increase in the bandwidth of the force amplitude.

\section{Damping of flexible structure}

The purpose of the numerical example in this section is both to illustrate the efficiency of the balanced calibration procedure with equal modal damping properties, and to demonstrate the importance of including the effect from non-resonant vibration modes via the new background flexibility parameter $\kappa_{0}$.

Figure 5 shows two identical piezoelectric laminate transducers placed symmetrically on a cantilever beam of length $10 a$. Because of the transducer symmetry the neutral beam axis is constant along the beam, and the two piezoelectric transducers are con-

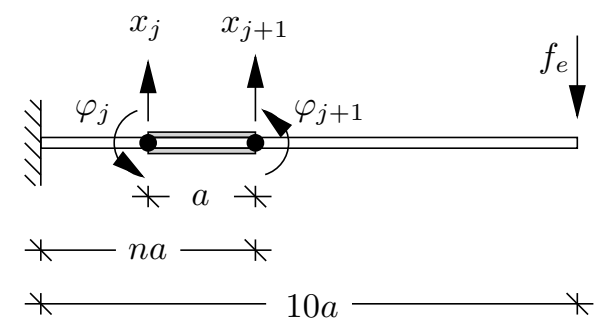

Figure 5: Cantilever beam with symmetric pair of piezoelectric laminate transducers. 
veniently treated as a single transducer couple with effective piezoelectric and shunt circuit parameters. The beam is discretized by 10 finite beam elements of length $a$ with transverse displacement $x_{j}$ and cross-section rotation $\varphi_{j}$ as the two degrees of freedom of node $j$. Thus, the discretization of the beam is chosen so that the pair of piezoelectric transducers occupies a single element $n$. The bending stiffness of the beam is $E I$, while the total contribution to the bending stiffness from the transducer couple with short-circuit electrodes is $E I_{p}=\frac{1}{2} E I$. The stiffness matrix is then represented by

$$
\mathbf{K}=\mathbf{K}_{b}+E I_{p} \mathbf{w} \mathbf{w}^{T}
$$

where $\mathbf{K}_{b}$ is the stiffness matrix of the beam without piezoelectric transducers, while the connectivity vector

$$
\mathbf{w}=[\ldots 0, \underbrace{0-1,0,1}_{\text {element } n}, 0 \ldots]^{T}
$$

defines the change in rotation $\varphi_{j+1}-\varphi_{j}=\mathbf{w}^{T} \mathbf{u}$ across element $n$ as the effective measure of deformation of the transducer couple. Thus, the contribution from local bending of the piezoelectric transducers is neglected in $\mathbf{K}$. Furthermore, the inertia of the transducers is not taken into account by the mass matrix $\mathbf{M}$.

The piezoelectric transducers are connected to individual resonant shunt circuits with identical resistance $R$ and inductance $L$. As demonstrated in (56) and (57) the dimensions and properties of the piezoelectric transducer are conveniently chosen or estimated so that a desired value of the modal damping ratio $\zeta=\zeta_{\text {des }}$ is obtained for the resonant vibration mode of the structure. The results from the calibration procedure are presented in Table 2 for the series shunt circuit and in Table 3 for the parallel shunt circuit. For both shunt circuit configurations the calibration is conducted for all four combinations of the desired damping ratios $\zeta_{\text {des }}=0.02$ and 0.04 and the transducer locations $n=2$ and 4 . These results are summarized in the top half of each table, while the bottom half presents the corresponding results for $\kappa_{0}=0$ without correction for background flexibility.

The modal stiffness $k_{r}$ is initially determined by (23) for the first vibration mode $(r=1)$ and the flexibility $1 / k_{0}$ from the non-resonant background modes subsequently 
Table 2: Complex root analysis: Series $R L$ shunt circuit.

\begin{tabular}{|c|c|c|c|c|c|c|c|c|}
\hline$n$ & $\zeta_{\mathrm{des}}$ & $\kappa_{r}$ & $\kappa_{0}$ & $L C \omega_{r}^{2}$ & $R C \omega_{r}$ & $\frac{\omega_{e}}{\omega_{r}}$ & $\zeta_{e}$ & $\zeta$ \\
\hline 2 & 0.02 & 0.0032 & 0.0145 & 0.9795 & 0.0785 & 1.0104 & 0.0397 & $\begin{array}{l}0.0200 \\
0.0200\end{array}$ \\
\hline & 0.04 & 0.0136 & 0.0605 & 0.9193 & 0.1480 & 1.0430 & 0.0772 & $\begin{array}{l}0.0400 \\
0.0400\end{array}$ \\
\hline 4 & 0.02 & 0.0033 & 0.0403 & 0.9551 & 0.0765 & 1.0232 & 0.0392 & $\begin{array}{l}0.0200 \\
0.0200\end{array}$ \\
\hline & 0.04 & 0.0151 & 0.1834 & 0.8238 & 0.1326 & 1.1018 & 0.0731 & $\begin{array}{l}0.0400 \\
0.0399\end{array}$ \\
\hline 2 & 0.02 & 0.0032 & 0.0000 & 0.9936 & 0.0796 & 1.0032 & 0.0399 & $\begin{array}{l}0.0237 \\
0.0166\end{array}$ \\
\hline & 0.04 & 0.0128 & 0.0000 & 0.9749 & 0.1570 & 1.0128 & 0.0795 & $\begin{array}{l}0.0549 \\
0.0273\end{array}$ \\
\hline 4 & 0.02 & 0.0032 & 0.0000 & 0.9936 & 0.0796 & 1.0032 & 0.0399 & $\begin{array}{l}0.0290 \\
0.0118\end{array}$ \\
\hline & 0.04 & 0.0128 & 0.0000 & 0.9749 & 0.1570 & 1.0128 & 0.0795 & $\begin{array}{l}0.0715 \\
0.0143\end{array}$ \\
\hline
\end{tabular}

follows from (24) as $1 / k_{0}=\mathbf{w}^{T} \mathbf{K}^{-1} \mathbf{w}-1 / k_{r}$. Based on the desired damping ratio $\zeta=\zeta_{\text {des }}$ the generalized coupling coefficient $\kappa_{r}$ is then determined by the design formula in (56), and the background flexibility parameter finally follows from (37) as $\kappa_{0}=$ $\kappa_{r} k_{r} / k_{0}$. The effective shunt circuit components $L C$ and $R C$ can now be determined in non-dimensional form by the design formulae in Table 1, while the corresponding filter components $\omega_{e}$ and $\zeta_{e}$ are given in (43), (46) for the series shunt circuit and in (52), (54) for the parallel shunt circuit.

It follows from Tables 2 and 3 that for the present cantilever beam example the background correction coefficient $\kappa_{0}$ is in fact significantly larger than the generalized coupling coefficient $\kappa_{r}$, and for the most indirect transducer location at $n=4$ the flexibility ratio $\kappa_{0} / \kappa_{r}=k_{r} / k_{0}>10$, which clearly identifies the importance of the $\kappa_{0}$ correction in the calibration expressions in Table 1. The efficiency of resonant damping 
Table 3: Complex root analysis: Parallel $R L$ shunt circuit.

\begin{tabular}{cccccccccc}
\hline$n$ & $\zeta_{\text {des }}$ & $\kappa_{r}$ & $\kappa_{0}$ & $L C \omega_{r}^{2}$ & $1 /\left(R C \omega_{r}\right)$ & $\frac{\omega_{e}}{\omega_{r}}$ & $\zeta_{e}$ & $\zeta$ \\
\hline 2 & 0.02 & 0.0032 & 0.0145 & 0.9857 & 0.0812 & 1.0072 & 0.0403 & 0.0200 \\
& & & & & & & & 0.0200 \\
& 0.04 & 0.0136 & 0.0605 & 0.9430 & 0.1697 & 1.0298 & 0.0824 & 0.0400 \\
4 & 0.02 & 0.0033 & 0.0403 & 0.9613 & 0.0832 & 1.0200 & 0.0408 & 0.0200 \\
& & & & & & & & 0.0200 \\
& 0.04 & 0.0151 & 0.1834 & 0.8450 & 0.1893 & 1.0878 & 0.0870 & 0.0400 \\
& & & & & & & & 0.0399 \\
\hline & 0.02 & 0.0032 & 0.0000 & 1.0000 & 0.0800 & 1.0000 & 0.0400 & 0.0233 \\
& & & & & & & & 0.0164 \\
& 0.04 & 0.0128 & 0.0000 & 1.0000 & 0.1600 & 1.0000 & 0.0800 & 0.0511 \\
& & & & & & & & 0.0266 \\
& 0.02 & 0.0032 & 0.0000 & 1.0000 & 0.0800 & 1.0000 & 0.0400 & 0.0276 \\
& & & & & & & & & 0.0117 \\
& 0.04 & 0.0128 & 0.0000 & 1.0000 & 0.1600 & 1.0000 & 0.0800 & 0.0601 \\
& & & & & & & & 0.0142 \\
\hline
\end{tabular}

concepts depends to a great extend on the accurate tuning of the filter frequency $\omega_{e}$, which is governed by the shunt circuit inductance $L$. By comparison of the cases with and without $\kappa_{0}$ in the tables it is found that the calibration with $\kappa_{0}=0$ overestimates the non-dimensional inductance $L C \omega_{r}^{2}$ by more than $18 \%$ for the case with $\zeta_{\text {des }}=0.04$ and $n=4$. The non-dimensional resistance $R C \omega_{r}$ governs the damping level of the shunt circuits, and the results in Tables 2 and 3 show that including the background correction factor $\kappa_{0}$ implies that the optimal non-dimensional resistance is reduced by up to $15 \%$ compared to the case with $\kappa_{0}=0$.

The last column in Tables 2 and 3 gives the damping ratios for the two modes associated with the first vibration form of the beam structure. The damping ratio is determined as $\zeta=\operatorname{Im}[\omega] /|\omega|$, where the complex-valued natural frequency $\omega$ is governed by the eigenvalue problem constituted by the homogeneous form of the structural 
equation of motion (15) together with the filter equation (3) for the series circuit or (10) for the parallel circuit. The results in the top half of both tables show that the two damping ratios are virtually identical and equal to the corresponding desired value $\zeta_{\text {des. }}$ This demonstrates that equal modal damping is obtained when using the present balanced calibration procedure with correction by the background flexibility factor $\kappa_{0}$. In the bottom half of the tables, with $\kappa_{0}=0$, the equal modal damping property is lost, as the damping ratio from the first eigenvalue becomes too large, while the damping ratio from the second eigenvalue is reduced, thereby limiting the effective damping of the resonant vibration mode.

Equal modal damping relies on the inverse relation (29) and is therefore by construction implied in the generic equations (30) and (31). It is a very desirable property of the balanced calibration procedure because it represents a system property that is independent of the particular loading and response conditions. In the generic equation in Section 4 the parameter $\lambda$ governs the damping level. As explained previously $\lambda=1$ corresponds to the bifurcation point $\omega_{b}$ in Fig. 3, which leads to the largest damping level within the equal damping regime. However, at this point undesirable interference occurs between the two modes, which again leads to a significant peak in the frequency response amplitude at resonance. In [26] it has been demonstrated for the mechanical tuned mass absorber that the intermediate level $\lambda=\frac{1}{2} \sqrt{2}$ leads to effective reduction of the vibration amplitude for both structure and absorber mass, and this design value is therefore also applied for the present piezoelectric damping problem.

Figure 6 shows the frequency amplitude of the transverse tip motion $u_{\text {tip }}$ of the cantilever beam in $(\mathrm{a}, \mathrm{b})$ and the amplitude of the piezoelectric force $f$ in $(\mathrm{c}, \mathrm{d})$. The results are obtained from the frequency representation of the equation of motion (15) with the transverse tip force introduced by the load vector

$$
\mathbf{f}_{e}=\left[0 \ldots, f_{e}, 0\right]^{T}
$$

and the corresponding filter equation (3) or (10). Because the dynamic tip motion amplitude is normalized by the corresponding static deflection $u_{\text {tip }}^{0}$ the curves in Fig. 6(a,b) represent the dynamic amplification, while the force in Fig. 6(c,d) is normalized by the modal load $f_{r}$ defined in (28b). Thus, the frequency amplitude curves in Fig. 6 repre- 
sent results that are directly comparable with those for the idealized single mode case in Fig. 4. Each of the sub-figures in Fig. 6 contain curves for the desired modal damping ratios $\zeta_{\text {des }}=0.02$ and 0.04 , as in Table 2 and Fig. 4 . The solid curves represent the case with correction for background flexibility, while the dashed curves represent the corresponding case with $\kappa_{0}=0$. The left sub-figures $(\mathrm{a}, \mathrm{c})$ contain the curves for the transducer location close to the support $n=2$, while the right pair of figures (b,d) correspond to the more indirect location at $n=4$. It is observed that the solid curves in Fig. 6 show both the desired reduction in the vibration amplitude of the structure and the entirely flat plateau in the magnitude of the piezoelectric force. In fact, the solid curves practically reproduce the results for the idealized case presented in Fig. 4, irrespective of the transducer location. For $n=2$ the dashed curves in Figs. 6(a,c) exhibit a visible detuning because $\kappa_{0}=0$. For the double peak plateau of the dynamic
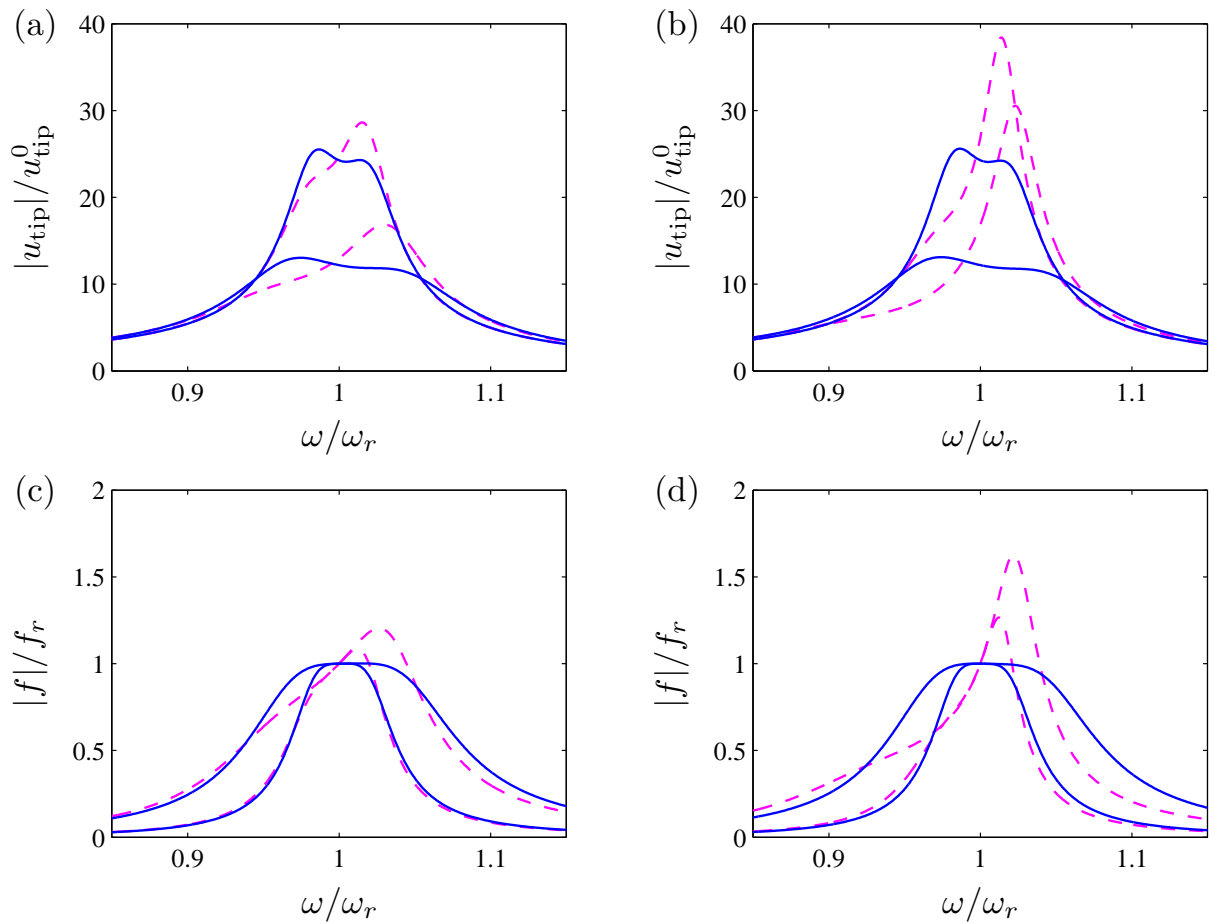

Figure 6: (a,b) Dynamic amplification and (c,d) force amplitude for series $R L$ shunt circuit with $n=2$ (a,c) and $n=4(\mathrm{~b}, \mathrm{~d})$. 

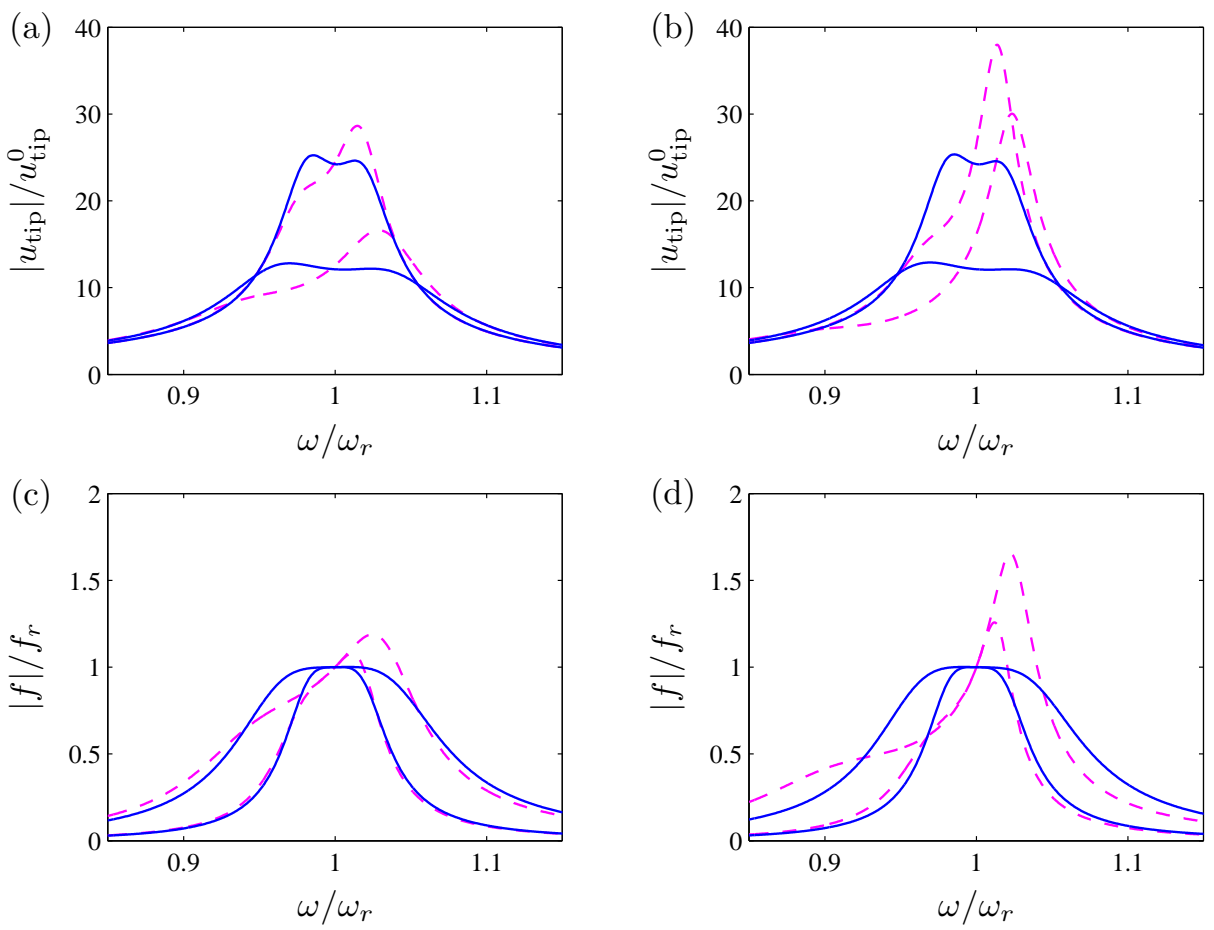

Figure 7: (a,b) Dynamic amplification and (c,d) force amplitude for parallel $R L$ shunt circuit with $n=2(\mathrm{a}, \mathrm{c})$ and $n=4(\mathrm{~b}, \mathrm{~d})$.

amplification in Fig. 6(a) the left peak is lowered, whereas the right peak has become larger. This corresponds well with the observed loss of equal modal damping in the bottom rows $\left(\kappa_{0}=0\right)$ of Table 2 , which is mainly due to the detuning of the shunt circuit inductance. For the more indirect location of the piezoelectric transducers at $n=4$ the dashed curves in Figs. $6(\mathrm{~b}, \mathrm{~d})$ present a severe increase of the amplitudes by approximately $50 \%$ compared to the calibration case where the $\kappa_{0}$ correction has been included. Figure 7 shows the corresponding results for the parallel shunt circuit, and the results and conclusions are very similar to those for the series configuration in Fig. 6 .

\section{Conclusions}

The design of piezoelectric $R L$ shunt circuits is commonly based on a single mode representation of the structural response, whereby the electromechanical system is gov- 
erned by two coupled scalar equations: The modal equation of motion and the corresponding electric filter equation [20]. This format implies that the electromechanical coupling coefficient can be identified directly from the natural frequencies associated with the short- and open circuit limits of the piezoelectric transducer, or from the frequency response properties of the structure when introducing a resonant shunt circuit $[38,39]$. Although the electromechanical coefficient may be determined accurately, the assumed single mode representation is still an approximation, and the theoretical predictions of the resistance $R$ and inductance $L$ are therefore often re-calibrated [8,9] to give the desired flat plateau in the frequency response curves. In particular the electric filter frequency must be calibrated precisely, and in $R L$ shunt circuits the inductance $L$ is conveniently adjusted by changing the variable components of the corresponding synthetic inductor [10, 20, 25].

In the present paper the deformation of the piezoelectric transducer, which in principle contains contributions from all vibration modes, is approximated by the resonant contribution from the targeted vibration mode together with the quasi-static contribution from the remaining non-resonant vibration modes. This representation introduces an additional background flexibility parameter $\kappa_{0}$, which modifies the coefficients of the electric filter equation. As demonstrated, it is straight forward to take the presence of the background flexibility parameter into account by the proposed balanced calibration procedure, because it is based on pole-placement by equivalence between the governing characteristic equation and a generic equation with implied equal modal damping. This procedure only involves the denominator of the frequency transfer function and not the numerator, and thus identical calibration results are obtained with respect to displacement (compliance), velocity (mobility) and acceleration, see [27]. The present balanced calibration procedure with explicit correction for background flexibility comprises a number of potential benefits: (a) equal modal damping, (b) effective reduction of the dynamic amplification, (c) no overshoot of piezoelectric force amplitude, and (d) explicit formulae for the system parameters in terms of structural modal properties and the desired damping ratio.

The accuracy of the balanced calibration procedure is illustrated by a simple numerical example. It is shown that the presence of the background flexibility parameter leads 
to a reduction in both inductance and resistance, compared to the calibration expressions with $\kappa_{0}=0$. Furthermore, it is seen that the property of equal modal damping requires the correction for background flexibility, and in the case without correction $\left(\kappa_{0}=0\right)$ the desired flat plateau of the double peaks in the dynamic amplification is lost, introducing a significant increase in the response amplitudes.

[1] S.O.R. Moheimani, A survey of recent innovations in vibration damping and control using shunted piezoelectric transducers, IEEE Transactions on Control Systems Technology, 11 (2003) 482-494.

[2] S.O.R. Moheimani, A.J. Fleming, Piezoelectric transducers for vibration control and damping, Springer, London, 2006.

[3] A. Preumont, Vibration Control of Active Structures. An Introduction, 3rd edition, Springer, Heidelberg, 2011.

[4] E.H. Anderson, N.W. Hagood, Simultaneous piezoelectric sensing/actuation: Analysis and application to controlled structures, Journal of Sound and Vibration 174 (1994) 617-639

[5] S.-M. Kim, S. Wang, M.J. Brennan, Dynamic analysis and optimal design of a passive and an active piezo-electrical dynamic vibration absorber, Journal of Sound and Vibration 330 (2011) 603-614.

[6] J. Ducarne, O. Thomas, J.-F. Deü, Structural vibration redduction by switch shunting of piezoelectric elements: Modeling and optimization, Journal of Intelligent Material Systems and Structures 21 (2010) 797-816.

[7] N.W. Hagood, A. von Flotow, Damping of structural vibrations with piezoelectric materials and passive electrical networks, Journal of Sound and Vibration 146 (1991) 243-268.

[8] J.J. Hollkamp, Multimodal passive vibration suppression with piezoelectric materials and resonant shunts, Journal of Sound and Vibration 146 (1991) 243-268. 
[9] S.Y. Wu, Piezoelectric shunts with a parallel R-L circuit for structural damping and vibration control, SPIE Proceedings 2720 (1996) 259-269.

[10] C.H. Park, D.J. Inman, A uniform model for series R-L and parallel R-L shunt circuits and power consumption, SPIE Proceedings, 3668 (1999) 797-804.

[11] A. Preumont, B. de Marneffe, A. Deraemaeker, F. Bossens, The damping of a truss structure with a piezoelectric transducer, Computers and Structures 86 (2008) 227239.

[12] C.L. Davis, G.A. Lesieutre, A modal strain energy approach to the prediction of resistively shunted piezoceramic damping, Journal of Sound and Vibration 184 (1995) $129-139$.

[13] D.A. Saravanos, Passively damped laminated piezoelectric shell structures with integrated electric networks, AIAA Journal 38 (2000) 1260-1268.

[14] O.M. Fein, A model for piezo-resistive damping of two-dimensional structures, Journal of Sound and Vibration 310 (2008) 865-880.

[15] Y. Liao, H.A. Sodano, Piezoelectric damping of resistively shunted beams and optimal parameters for maximum damping, Journal of Vibration and Acoustics 132 (2010) 041014 (7pp).

[16] R.L. Forward, Electronic damping of vibrations in optical structures, Applied Optics 18 (1979) 690-697.

[17] J. Tang, K.W. Wang, Active-passive hybrid piezoelectric networks for vibration control: Comparisons and inprovement, Smart Materials and Structures 10 (2001) $794-806$.

[18] B. de Marneffe, A. Preumont, Vibration damping with negative capacitance shunts: theory and experiments, Smart Materials and Structures 17 (2008) 035015 (9pp).

[19] S. Manzoni, S. Moschini, M. Redaelli, M. Vanali, Vibration attenuation by means of piezoelectric transducer shunted to sunthetic negative capacitance, Journal of Sound and Vibration 331 (2012) 4644-4657. 
[20] O. Thomas, J. Ducarne, J.-F., Deü, Performance of piezoelectric shunts for vibration reduction, Smart Materials and Structures, 80 (2012) 235-268.

[21] J.E. Brock, A note on the damped vibration absorber, Journal of Applied Mechanics 13 (1946) A284.

[22] J.P. Den Hartog, Mechanical Vibrations, 4th edition, McGraw-Hill, New York, 1956.

[23] G. Caruso, A critical analysis of electric shunt circuits employed in piezoelectric passive vibration damping, Smart Materials and Structures 10 (2001) 1059-1068.

[24] K. Yamada, H. Matsuhisa, H. Utsuno, K. Sawada, Optimum tuning of series and parallel LR circuits for passive vibration suppression using piezoelectric elements, Journal of Sound and Vibration 329 (2010) 5036-5057.

[25] M.V. Kozlowski, D.G. Cole, R.L. Clark, A comprehensive study of the RL series resonant shunted piezoelectric: A feedback controls perspective, Journal of Vibration and Acoustics 133 (2011) 011012 (10pp).

[26] S. Krenk, Frequency analysis of the tuned mass damper, Journal of Applied Mechanics 72 (2005) 936-942.

[27] S. Krenk, J. Høgsberg, Equal modal damping design for a family of resonant vibration control formats, Journal of Vibration and Control 19 (2013) 1294-1315.

[28] J. Høgsberg, S. Krenk, Balanced calibration of resonant shunt cicuits for piezoelectric vibration control, Journal of Intelligent Material Systems and Structures 23 (2012) 1937-1948.

[29] R.L. Bisplinghoff, H. Ashley, Principles of Aeroelasticity, Wiley, New York, 1962.

[30] O.E. Hansteen, K. Bell, Accuracy of mode superposition analysis in structural dynamics, Earthquake Engineering and Structural Dynamics 7 (1979) 405-411.

[31] R.L. Clark, Accounting for out-of-bndwidth modes in the assumed modes approach: Implications on colocated output feedback control, Journal of Dynamic Systems, Measurement, and Control 119 (1997) 390-395. 
[32] S. Krenk, J. Høgsberg, Optimal resonant control of flexible structures, Journal of Sound and Vibration 323 (2009) 530-554.

[33] M.N. Svendsen, S. Krenk, J. Høgsberg, Resonant vibration control of rotating beams, Journal of Sound and Vibration 330 (2011) 1877-1890.

[34] S. Krenk, M.N. Svendsen, J. Høgsberg, Resonant vibration control of three-bladed wind turbine rotors, AIAA Journal 50 (2012) 148-161.

[35] S. Krenk, J. Høgsberg, Tuned mass absorber on a flexible structure, Journal of Sound and Vibration 333 (2014) 1577-1595.

[36] H.H. Law, P.L. Rossiter, G.P. Simon, L.L. Koss, Characterization of mechanical vibration damping by piezoelectric materials, Journal of Sound and Vibration 197 (1996) 489-513

[37] A. Belloli, D. Niederberger, S. Pietrzko, M. Morari, P. Ermanni, Structural vibration control via R-L shunted active fiber composites, Journal of Intelligent Material Systems and Structures 18 (2007) 275-287.

[38] M. Porfiri, C. Maurini, J. Pouget, Identification of electromechanical modal parameters of linear piezoelectric structures, Smart Materials and Structures 16 (2007) 323-331.

[39] T. Delperro, A.E. Bergamini, P. Ermanni, Identification of electromechanical parameters in piezoelectric shunt damping and loss factor prediction, Journal of Intelligent Material Systems and Structures 24 (2013) 287-298 